\title{
Generation of optical absorption band in colloidal coffee at extremely alkaline $\mathrm{pH}$
}

\author{
Arindam Kushagra ${ }^{1}$ and Akshaya Pandey ${ }^{2}$ \\ ${ }^{1}$ Amity Institute of Nanotechnology, Amity University, Kolkata 700135, INDIA \\ ${ }^{2}$ Amity Institute of Biootechnology, Amity University, Kolkata 700135, INDIA
}

\begin{abstract}
Coffee and caffeine have been used as solar absorbers and also to increase the thermal stability and efficiency of perovskite solar cells. In this work, we report the sensing of extremely alkaline pH by colloidal coffee solution aided by generation of an optical absorption band in the near-UV region. This generation of absorption band could be explained by the orientation induced dipole-dipole interactions arising from differing caffeine-solvent interactions with varying $\mathrm{pH}$. Such a generation leads to the lowering of direct as well as indirect bandgaps from 4 $\mathrm{eV} \rightarrow 2.8 \mathrm{eV} \& 3.4 \mathrm{eV} \rightarrow 2.5 \mathrm{eV}$, respectively. We also estimate the changes in optical energy storage efficiency, inferring it to be highest for $\mathrm{pH} 11$ having the highest intensity of the generated absorption band $\left(\lambda_{\text {abs }} \approx 360 \mathrm{~nm}\right)$. With these observations and further deductions, the work reported in this paper would be of immense interest to the researchers working in the field of development of chemical pH sensors and also in the development of novel UV absorbers.
\end{abstract}

Index Terms-Caffeine, colloidal coffee, optical absorption, pH sensor, solute-solvent interactions, UV absorber

\section{INTRODUCTION}

Coffee is one of the most consumed beverage around the world [1].Its primary constituent is caffeine, which stimulates the central nervous system [2] and is the world's most widely consumed psychoactive substance that is legally permissible [3]. Recently, coffee and its ingredient caffeine have garnered attention of researchers working in the field of photovoltaics. Colloidal solution of coffee has been shown to be useful as a material for direct solar absorption [4] whereas caffeine has been used to improve the performance and thermal stability of perovskite solar cells [5]. Recently, an alternative way to sense extremely alkaline $\mathrm{pH}$ using the shape of hydrophobe-water interfaces was reported [6].

In this paper, we report the sensing of extremely alkaline $\mathrm{pH}(11 \leq$ $\mathrm{pH} \leq 13)$ aided by generation of an optical absorption peak $\left(\lambda_{\mathrm{abs}} \approx\right.$ $360 \mathrm{~nm}$ ) in the near-UV region when the $\mathrm{pH}$ of the colloidal coffee solution was within the stipulated range. However when the $\mathrm{pH}$ of colloidal solution was less than or equal to 10 , the aforementioned absorption peak was not found. Henceforth we infer that colloidal coffee has an indirect optical bandgap, which can be further modified to a lower value by the generation of a new absorption band by changing the $\mathrm{pH}$ of colloidal coffee. The same phenomenon can be depicted schematically with the aid of an E-k diagram, as shown in Fig. 1.
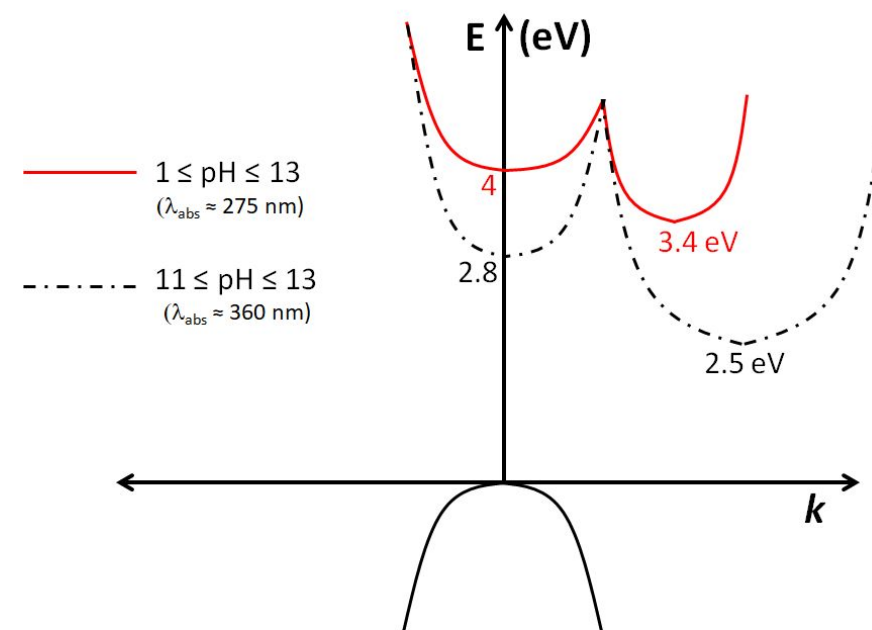

Fig. 1E- $\boldsymbol{k}$ diagram of colloidal coffee solution showing the lowering of optical bandgap due to generation of an optical absorption peak $\left(\lambda_{a b s} \approx 360 \mathrm{~nm}\right)$ denoted by dashed line.

The coordinates in Fig. 1 lying on the y-axis (i.e. $k=0$ ) denote the values of direct bandgap and indirect bandgap is shown for values $\boldsymbol{k} \neq 0$ [7]. Details of the same are discussed further in the paper. In continuation to the earlier discussion, since coffee and its ingredient caffeine have now gained attention of researchers working in the field of solar photovoltaics, this work would be of immense interest to the same group especially to those working on the bandgap engineering and further development of novelUV absorbers.

\section{MATERIALS AND METHODS}

Commercially available instant coffee Nescafe Classic was used in this work. Estimated caffeine content in such products is $\approx 26$ $\mathrm{mg} / \mathrm{g}$ of instant coffee [8]. A stock colloidal solution of $1 \mathrm{mg} / \mathrm{ml}$ was prepared by mixing instant coffee with Milli-Q water. $0.1 \mathrm{ml}$ of the colloidal coffee was taken from the prepared stock solution and mixed with $2.6 \mathrm{ml}$ of Milli-Q water and $0.3 \mathrm{ml}$ of aqueous solutions

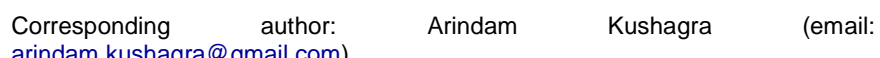
arindam.kushagra@gmail.com). 
of varying $\mathrm{pH}$ (pH: 0-14) prepared by serial dilution. For the acidic phase, 35\% $\mathrm{HCl}$ (Emplura Merck, 1.93401.0521) was taken as stock solution. Normality of the stock $\mathrm{HCl}$ solution was calculated to be $\approx$ 11.33. Since $\mathrm{HCl}$ is a monoprotic acid, the molarity would be same as that of normality i.e. $\approx 11.33 \mathrm{M}$. Suitable dilutions of the same were done to obtain solutions in the range of $\mathrm{pH} 0(1 \mathrm{M})$ till $\mathrm{pH} 6$ $\left(10^{-6} \mathrm{M}\right)$. Milli-Q water was taken as a neutral standard. For the alkaline phase, $1 \mathrm{M} \mathrm{NaOH}$ solution was prepared as stock using $\mathrm{NaOH}$ pellets (Himedia, MB095). Serial dilutions of $\mathrm{NaOH}$ stock were done to obtain solutions in the range $\mathrm{pH} 8\left(10^{-6} \mathrm{M}\right)$ till $\mathrm{pH} 13$ $\left(10^{-1} \mathrm{M}\right)$. Optical absorbance and transmittance measurements were done using U-2910 Hitachi spectrophotometer.

\section{RESULTS AND DISCUSSION}

Optical absorbance spectra for different $\mathrm{pH}$ values of colloidal coffee solution, showing the unique absorption peaks at $360 \mathrm{~nm}$ are evident at $\mathrm{pH}$ values 11-13 from the following figure, Fig. 2:

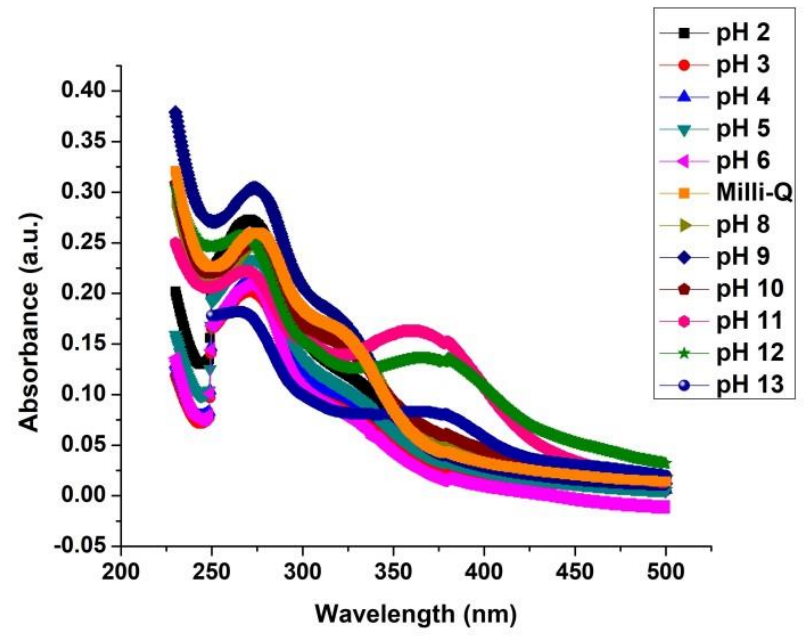

Fig. 2Optical absorption peaks of colloidal coffee solution at various $\mathrm{pH}$ values, ranging from 2-13, showing distinct peaks @ 360 $\mathrm{nm}$ for $\mathrm{pH}$ values 11-13 only.

Absorption coefficient $(\alpha)$ was calculated using the relationship: $\alpha(T)=-\frac{1}{l} \ln \left(\frac{T}{\left(1-R^{2}\right)}\right)$ [9]. Here, $l$ is the path length traversed by light during absorption/transmission measurements (taken to be $1 \mathrm{~cm}$ ), $T$ is the measured transmittance and $R$ is reflectance. Since the measurements in this work were carried out in liquid phase, reflectance would be negligible i.e. $R=0$. Direct and indirect bandgaps were calculated by Tauc plot analysis i.e. by plotting $(\alpha h v)^{2} \& \quad(\alpha h v)^{0.5}$ vs. $h v$ (energy of the measured electromagnetic spectrum, in eV), respectively [10]. The calculated direct and indirect bandgaps are shown in following figures $3 \& 4$ :

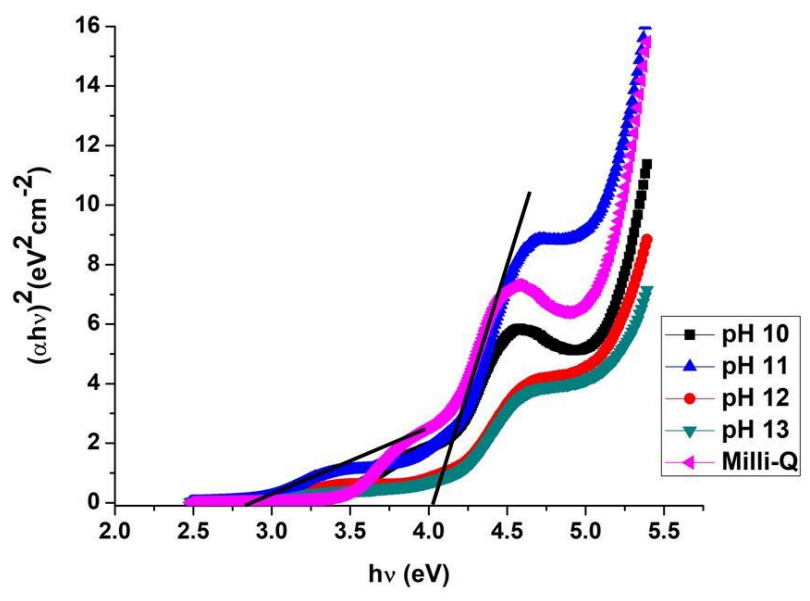

Fig. 3 Calculation of direct bandgaps of colloidal coffee solutions at $\mathrm{pH} 10,11,12,13$ and at neutral $\mathrm{pH}$ (represented by Milli-Q water) using Tauc-plot method, showing bandgap $\approx 4 \mathrm{eV}$ for $\lambda_{\mathrm{abs}} \approx$ $275 \mathrm{~nm} \&$ bandgap $\approx 2.8 \mathrm{eV}$ for $\lambda_{\text {abs }} \approx 360 \mathrm{~nm}$, respectively.

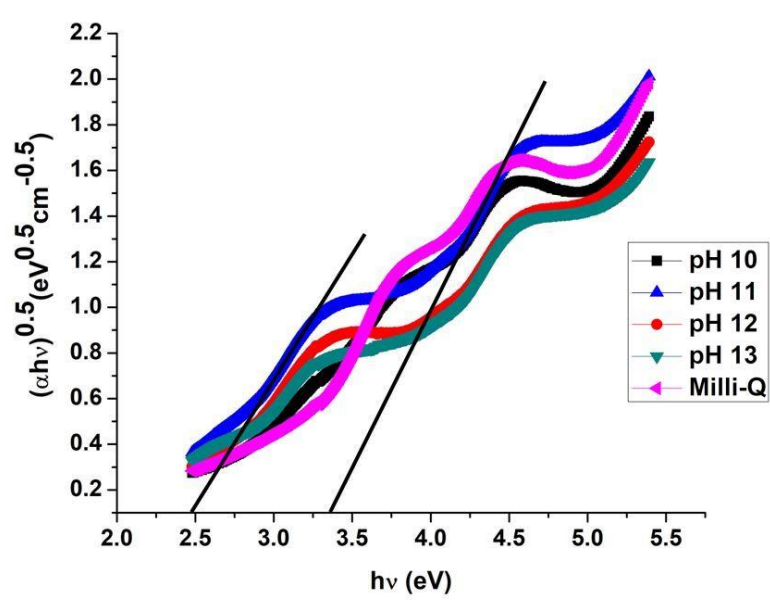

Fig. 4.Calculation of indirect bandgaps of colloidal coffee solutions at $\mathrm{pH} 10,11,12,13$ and at neutral $\mathrm{pH}$ (represented by Milli-Q water) using Tauc-plot method, showing bandgap $\approx 3.4 \mathrm{eV}$ for $\lambda_{\mathrm{abs}} \approx 275 \mathrm{~nm} \&$ bandgap $\approx 2.5 \mathrm{eV}$ for $\lambda_{\mathrm{abs}} \approx 360 \mathrm{~nm}$, respectively.

Generation of the said optical absorption band can be attributed to the solute-solvent interactions in colloidal coffee. These solutesolvent interactions can be described by the following analytical relationships [11], as discussed in the following equations, 1-3.

$$
\begin{aligned}
h c \Delta v_{\text {ind }}=-\frac{2 n^{2}+1}{n^{2}+2} & \left\{\frac{2 \mu_{g}\left(\mu_{g}-\mu_{e} \cos \theta\right)}{a^{3}}\left[\frac{\epsilon-1}{\epsilon+2}-\frac{n^{2}-1}{n^{2}+2}\right]\right. \\
+ & \left.\frac{\left(\mu_{g}-\mu_{e}\right)^{2}}{a^{3}} \frac{n^{2}-1}{n^{2}+2}\right\}
\end{aligned}
$$

where $\Delta v_{\text {ind }}=v_{o}-v_{s}$ is the change in wavenumber due to induced dipole moments accounting for solute-solvent interactions, $v_{o}$ is the wavenumber for isolated molecule in vapor phase (in this case, caffeine) $\& v_{s}$ is the wavenumber of caffeine molecule in solution. 
$n \& \epsilon$ are solvent refractive index \& dielectric constant, respectively. $a$ is the molecular radius of caffeine. $\mu_{g} \& \mu_{e}$ are induced dipole moments in the ground $\&$ excited states and $\theta$ is the angle between $\mu_{g} \& \mu_{e}$ vectors. There can be different types of dipole-dipole interactions that might impact the generation of the absorption band, which can be classified into orientation interactions \& dispersion force mediated interactions, respectively. As the name suggests, the orientation interactions represent the interactions of dipoles based on their orientations, as given by the relationship shown in equation 2 .

$$
h c \Delta v_{o r}=-\frac{h c^{2} f}{8 \pi m_{e} v_{o} a^{3}} \frac{n^{2}-1}{n^{2}+2}
$$

where $f(\epsilon)=\frac{\epsilon-1}{2 \epsilon+1} \& f(n)=\frac{n^{2}-1}{2 n^{2}+1}$ and $m_{e}$ is the mass of an electron. The orientation interaction mediated wavenumber shift is affected by the increasing polarity of the solvent. The dispersion force mediated interactions happen because of the electronic structure deformations of solute and solvent leading to the generation of small temporary dipole moments. Analytically, these interactions can be represented by the relationship shown in equation 3 .

$$
h c \Delta v_{\text {disp }}=\left(\alpha_{g}-\alpha_{e}\right) \frac{3}{2 a^{3}} \frac{I I^{\prime}}{I+I^{\prime}} \frac{n^{2}-1}{n^{2}+2}
$$

where $\alpha_{g} \& \alpha_{e}$ are polarizabilities of caffeine molecule in ground and excited states after interaction with the solvent medium. I\&I' represent the ionizing potentials in the ground and excited states, respectively. The dispersion force mediated interactions have a mere additive effect that is primarily impacted by the refractive index, $n$, of the solvent. On the basis of discussions made in context of equations $2 \& 3$, we deduce that the main reason of generation of the optical absorption band at $\lambda_{\text {abs }} \approx 360 \mathrm{~nm}$ is because of the orientation interactions between caffeine and aqueous solvents at $\mathrm{pH}$ 11-13. Since these solvents are extremely polar in nature, the electronic structure deformation of the caffeine-base complex formed from the solute-solvent interaction would cause the temporary dipole generation that would eventually lead to the generation of the said optical absorption band.

We also estimated the absorptive optical energy storage efficiency by first normalizing the transmittance values, $T=e^{-\alpha_{\lambda} l}\left(\alpha_{\lambda}\right.$ : wavelength-dependent absorption coefficient), from 0 to 1 . After normalizing, the transmittance values have been subsequently put into the following relationship that yields the energy storage efficiency [4] shown in equation 2:

$$
\eta=1-\frac{\int_{\lambda_{\min }}^{\lambda_{\max }} I_{\lambda} e^{-\alpha_{\lambda} l} d \lambda}{\int_{\lambda_{\min }}^{\lambda_{\max }} I_{\lambda} d \lambda}
$$

where $I_{\lambda}$ is the wavelength-dependent intensity of incident radiation and we also assume that there is no scattering of light from the colloidal sample. Since the measurements were done using a conventional spectrophotometer in laboratory settings, $I_{\lambda}$ can be assumed to be constant, equalling to the intensity of the lamp of spectrophotometer. The integrals given in equation 4 were solved numerically using composite trapezoidal rule [12]. The same is shown in Fig. 5, with an evident increase in optical energy storage efficiency for the generated absorption band at $360 \mathrm{~nm}$ in the $\mathrm{pH}$ range 11-13.

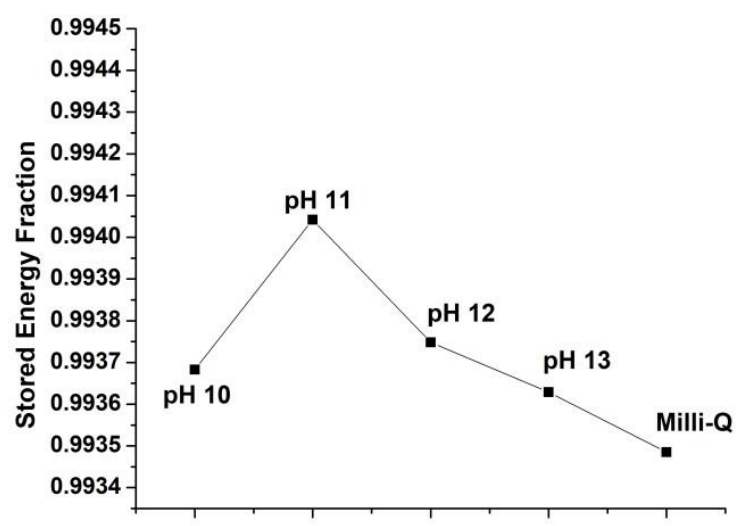

Fig. 5. Optical energy stored for varying $\mathrm{pH}$ values is shown. The increase in stored energy can be attributed to the generation of absorption band at $\approx 360 \mathrm{~nm}$, with intensities varying with $\mathrm{pH}$ in the order $11>12>13$.

\section{CONCLUSIONS}

In this work, we have reported the sensing of extremely alkaline $\mathrm{pH}$ with the aid of colloidal coffee solution and also observed the generation of an optical absorption band with an indirect bandgap of $\sim 2.5 \mathrm{eV}$. As discussed earlier in the paper, coffee and its primary ingredient caffeine have found applications as solar absorbers as well as in increasing the stability \& efficiency of perovskite solar cells. The results reported in this paper pave way towards the development of novel chemical sensors for extreme alkaline $\mathrm{pH}$ based on the spectrophotometric detection. This work could also be potentially used to develop ultraviolet light absorbers with increased capacity in optically absorbed energy storage in the near-UV range of the electromagnetic spectrum, actuated by modulating $\mathrm{pH}$ values of the colloidal coffee solution.

\section{ACKNOWLEDGMENT}

The authors acknowledge Dr. R.R. Bhattacharjee, Head, Amity Institute of Nanotechnology, Amity University Kolkata, for fruitful discussions and insights during the course of this work.

\section{REFERENCES}

[1]. 9 Most Consumed Beverages Around the World, http://www.haleysdailyblog.com/9-most-consumed-beverages-around-the-world/, accessed on $16^{\text {th }}$ June, 2019.

[2]. A. Nehlig, J-L. Daval, G. Debry (1992), Caffeine and the central nervous system: mechanisms of action, biochemical, metabolic and psychostimulant effects, Brain Res Rev., vol. 17, pp. 139-170.

[3]. J.W. Daly, J. Holmen, B.B. Fredholm (1998), Is caffeine addictive? The most widely used psychoactive substance in the world affects same parts of the brain as cocaine (in Swedish), Lakartidningen, vol. 95, pp. 5878-83.

[4]. M. Alberghini, M. Morciano, L. Bergamasco, M. Fasano, L. Lavagna, G. Humbert, E. Sani, M. Pavese, E. Chiavazzo, P. Asinari (2019), Coffee-based colloids for direct solar absorption, Sci. Rep., vol. 9, Article number: 4701. doi: https://doi.org/10.1038/s41598-019-39032-5

[5]. R. Wang, J. Xue, L. Meng, J-W Lee, Z. Zhao, P. Sun, L. Cai, T. Huang, Z. Wang, Z-K. Wang, Y. Duan, J. L. Yang, S. Tan, Y. Yuan, Y. Huang, Y. Yang (2019), Caffeine Improves the Performance and Thermal Stability of Perovskite Solar Cells, Joule, vol. 3, pp. 1-14.doi:https://doi.org/10.1016/j.joule.2019.04.005 
[6]. A. Kushagra, A.K. Pradhan, D. Bazal (2019), pH-dependent electrochemomechanical transition of hydrophobe-water interface, IEEE Sens. Lett., vol. 3, no. 7, 4500804. doi: https://doi.org/10.1109/LSENS.2019.2921146

[7]. k-space diagram, https://people.eecs.ku.edu/ demarest/470/KSpace\%20diagrams.pdf, accessed on $16^{\text {th }}$ June, 2019.

[8]. Beverages, coffee, instant, regular, prepared with water, https://fdc.nal.usda.gov/fdcapp.html\#/food-details/174130/nutrients, accessed on $23^{\text {rd }}$ June, 2019.

[9]. G. Benno, K. Joachim (2003), Optical Properties of Thin Semiconductor Films, http://home.fnal.gov/ jkopp/tum/pdf/F/hl_spekt.pdf , accessed on 16 $6^{\text {th }}$ June, 2019.

[10]. D. Suresh (2015), An Investigation on the Band Gap and Band Edge of SemiConducting Lanthanum Transition Metal Perovskites for Photocatalytic Applications, M.S. dissertation, University of South Florida.

[11]. G. Oanca, C. Nadejde, D. Creanga (2014), Caffeine-solvent interaction studied by UV-spectrometry and molecular modelling, Romanian J. Biophys., vol. 24, pp. 11-23.

[12]. Module for The Trapezoidal Rule for Numerical Integration, http://mathfaculty.fullerton.edu/mathews/n2003/TrapezoidalRuleMod.html, accessed on $21^{\text {st }}$ June, 2019. 